\title{
Bertolt Brecht und die Naturheilkunde
}

\author{
Claus Ruda \\ Berlin, Deutschland
}

\section{Schlüsselwörter}

Funktionelle Therapie - Immunsystem · Antibiotika · Unterstützung · Ersatzleistung $\cdot$ Naturheilkunde

\section{Zusammenfassung}

Jedes Organ des Körpers bedarf der ständigen Tätigkeit (Funktion). Wird seine Eigenleistung laufend von aussen zugeführt und ihm die Funktion abgenommen, so kommt es zur Untätigkeitsverkümmerung. Im Sinne einer funktionellen Therapie spricht man von Ersatzleistung. Was Brecht beispielhaft am Bewegungsapparat zeigt, gilt jedoch für alle Körperfunktionen. So wird heute oft vergessen, dass die Therapie mit Antibiotika für das Immunsystem eine Ersatzleistung ist. Die Folgen einer unkritischen Antibiotikatherapie werden beschrieben. Desgleichen ist eine bequeme Arzneitherapie für viele Kranke eine Krücke anstelle der notwendigen, aber unbequemen Änderung ihres Lebensstils. Nicht nur in der Medizin, sondern in vielen Lebensbereichen werden mit Ersatzleistungen die Eigenfunktionen eingeschränkt. Hier dürfte Brecht auch seine Erfahrungen gesammelt haben. Die Notwendigkeit einer «Funktionellen Therapie» wird betont. Ihre Grössen, mit denen gearbeitet werden muss, sind: Funktionsreiz, Organ, Funktion und Leistung; ihre Therapieformen: Ersatzleistung, Schonung, Übung und Unterstützung. Das Hauptanliegen der Naturheilkunde ist die Unterstützung der Eigenfunktionen, die Hilfe zur Selbsthilfe. Für jede Ersatzleistung gilt die strenge Indikation: So wenig wie möglich - so viel wie nötig. Ziel einer Behandlung soll immer der gesunde, autonome Mensch sein, der keine ärztliche Behandlung benötigt und ohne Krücken auskommt. Angesichts des Vorherrschens der Arzneitherapie, die weiter ausgebaut wird, haben viele Menschen heute andere Motive, den Arzt aufzusuchen, als Brechts Kranker.

\section{Keywords}

Functional therapy · Immune system · Antibiotics · Support . Substitution $\cdot$ Natural Medicine

\section{Summary}

\section{Bertolt Brecht and Natural Medicine}

Every organ in the body requires continuous activity. If the body's functioning is constantly being compensated from the outside and thereby relieved of its need to function, then a loss by disuse will occur. In the sense of functional therapy, one speaks in this case of substitute therapy. What Brecht points out in the example of the crutches for the musculoskeletal system is applicable to all body functions. Nowadays we often overlook the fact that antibiotics are a substitute for a healthy immune system - with negative results. Not only in medicine but also in many areas of life substitution limits the effectiveness of one's ability to function. The importance of functional therapy must therefore be emphasized. The main concern of natural medicine is to support, to enhance the body's functioning, and to help the body to help itself. A strict indication applies to every form of substitution. The aim of treatment should always be a healthy, autonomous individual who is able to cope without medical treatment or crutches. In view of the medical treatment prevailing today, which is focused on substituting instead of supporting the body's natural functioning, many people may well have other motives to see a doctor than Brecht's patient did.

\section{Mots-clés}

Thérapie fonctionnelle - Système immunitaire · Antibiotiques . Soutien · Substitution $\cdot$ Naturopathie

\section{Résumé}

Bertolt Brecht et la naturopathie

Chaque organe du corps nécessite un niveau continu d'activité (fonction). Si son activité est constamment compensée par des facteurs externes et sa fonction retirée, une atrophie liée à l'inactivité apparaît. Dans le contexte d'une thérapie fonctionnelle, on parle alors de substitution. Ce que Brecht a par exemple montré pour le système moteur s'applique à toutes les fonctions physiologiques. On oublie ainsi souvent aujourd'hui qu'un traitement antibiotique est un substitut du système immunitaire. Et les conséquences négatives d'un traitement antibiotique non justifié ont été décrites. De même, un traitement médicamenteux facile constitue pour de nombreux patients une béquille leur évitant un changement de style de vie nécessaire, mais désagréable. Des substituts viennent limiter les fonctions propres à l'organisme non seulement en médecine, mais aussi dans de nombreux autres domaines. Là-aussi, Brecht a pu en faire l'expérience. La nécessité d'une «thérapie fonctionnelle» est mise en relief. Les paramètres avec lesquels il faut travailler sont les suivants: stimulus fonctionnel, organe, fonction et performance. Les formes thérapeutiques sont:

\section{KARGER \\ Fax +497614520714 Information@Karger.d} www.karger.com
Dr. Claus Ruda

Bamberger Strasse 8, 10777 Berlin, Deutschland

Tel. +49 302183458

C.Ruda@T-Online.de 
substitution, protection, exercice et soutien. L'objectif principal de la naturopathie est de soutenir les fonctions propres à l'organisme et de favoriser l'effort personnel. À chaque substitution est associée la règle stricte «aussi peu que possible - autant que nécessaire». Un traitement doit toujours avoir pour objectif un être humain autonome et en bonne santé qui n'a besoin d'aucun traitement médical et s'en sort sans béquilles. Étant donnée la prédominance des traitements médicamenteux, qui continuent de se développer, de nombreuses personnes trouvent aujourd'hui bien d'autres raisons de consulter un médecin que celles du patient de Brecht.

Als der bayerische Arzt Lorenz Gleich 1849 das Wort «Naturheilkunde» prägte, war die wechselvolle Geschichte von Wort und Begriff nicht vorhersehbar. Ebenso wenig aber konnte man ahnen, dass 150 Jahre später ein Dichter mit psychologischem Einfühlungsvermögen die Naturheilkunde auf ihr theoretisches Konzept hinweisen würde.

Fragt man heute nach der Bedeutung des Wortes, so hört man unterschiedliche Antworten. In der Tat ist das, was sich unter dem Dach «Naturheilkunde» zu Recht oder zu Unrecht angesammelt hat, wesentlich umfangreicher und mit seiner ursprünglichen Bedeutung kaum vergleichbar, denn zuerst war sie nur Hydrotherapie [1]. 1903 war Naturheilkunde laut Meyers Lexikon «die Lehre von der Heilung der Krankheiten ohne Arzneien» [2]. Die offizielle Zeitschrift der Naturheilkunde hiess Der Naturarzt, Zeitschrift des Bundes der Vereine für Gesundheitspflege und für arzneilose Heilweise [3]. Um die Verbindung von Brecht zur Naturheilkunde zu finden, sind einige Vorbemerkungen erforderlich: Krankheiten gibt es, solange die Menschheit existiert, und ein Überleben war nur möglich, indem sich der Mensch die Fähigkeit zur Krankheitsbewältigung immer wieder aneignete. Knochenbrüche und Wunden gab es immer, ebenso Parasiten, mit denen sich der Mensch auseinandersetzen und an die er sich anpassen musste.

Hatte ihm die Stammesgeschichte keine angeborenen Fähigkeiten dazu mitgegeben, so legte sie ihm die Möglichkeit zur individuellen Anpassung in die Wiege. Die Selbstheilungskräfte des Körpers waren seit je bekannt und wurden nur unterschiedlich benannt.

Unabhängig von den angeborenen und erworbenen Heilungskräften des Körpers haben Ärzte immer wieder in den Heilungsprozess eingegriffen, um eine Krankheit überhaupt - oder besser - heilen zu können. Die jeweiligen wissenschaftlichen Erkenntnisse brachten dabei viele Erfolge, aber auch Misserfolge. Von einer Ursache für Misserfolge soll hier die Rede sein. Sie besteht darin, dass Ärzte glaubten, sie könnten den Selbstheilungskräften des Körpers ohne zwingenden Grund die Arbeit abnehmen. Damit aber verstiessen sie gegen das Grundgesetz der Physiologie und Pathophysiologie, das lautet: Jedes Organ bedarf der ständigen Tätigkeit, d.h. Funktion, wenn es nicht verkümmern soll. Hier wird der Begriff «Funktion» eingeführt, und hier soll Brecht [4] zu Worte kommen:

\author{
Die Krücken \\ Sieben Jahre wollt kein Schritt mir glücken. \\ Als ich zu dem großen Arzte kam \\ Fragte er: Wozu die Krücken? \\ Und ich sagte: Ich bin lahm. \\ Sagte er: Das ist kein Wunder. \\ Sei so freundlich, zu probieren! \\ Was dich lähmt ist dieser Plunder. \\ Geh, fall, kriech auf allen vieren! \\ Lachend wie ein Ungeheuer \\ Nahm er mir die schönen Krücken \\ Brach sie durch auf meinem Rücken \\ Warf sie lachend in das Feuer. \\ Nun, ich bin kuriert: ich gehe. \\ Mich kurierte ein Gelächter. \\ Nur zuweilen, wenn ich Hölzer sehe \\ gehe ich für Stunden etwas schlechter.
}

Brecht hat hier eindrucksvoll die Folgen beschrieben, die entstehen, wenn einem Organ, hier dem Bewegungsapparat, die Funktion abgenommen und dessen Leistung ständig durch Hilfe von aussen ersetzt wird: Die Eigenleistung verkümmert bis zum Erlöschen. Die Krücken stehen hier beispielhaft für alle Funktionen des Körpers, die Inaktivitätsatrophie nur als ein sichtbares Zeichen. In einem System der Therapie, für das sich der Terminus «Funktionelle Therapie» anbietet, wird dieses therapeutische Prinzip als «Ersatzleistung» bezeichnet, bekannt als Substitution bei inneren Organen.

Die medizinische Theorie der Pathologie wurde durch von Bergmann zur «Funktionellen Pathologie» erweitert [5]. In der Diagnostik ist die «Funktionelle Diagnostik» allgemein üblich. Lediglich in der Therapie hat sich das Denken in funktionellen Zusammenhängen - aus verschiedenen Gründen - nicht durchsetzen können. Wie an anderer Stelle ausführlicher beschrieben [6], sei hier nur kurz darauf eingegangen: Jedes Organ benötigt seinen Funktionsreiz. Das Ergebnis der Funktion ist die Leistung. Damit sind die Grössen genannt, mit denen die Funktionelle Therapie arbeitet. Dies sind: Funktionsreiz, Organ, Funktion, Leistung; und als Therapie: Ersatzleistung, Schonung, Übung; und übergeordnet: Unterstützung. Alle Prinzipien sind in der Medizin heute üblich; das therapeutische Prinzip der Naturheilkunde ist die Unterstützung.

In einem Buch der Naturheilheilverfahren wird man das Wort «Antibiotikum» nicht finden, denn es ist eine Ersatzleistung; es ist eine Krücke für das Immunsystem. Obwohl kein Arzt die oft lebensrettende Gabe eines Antibiotikums oder die Insulininjektion vermeiden würde, versucht die Naturheilkunde zunächst ohne Krücken auszukommen und den Körper in seinem Bemühen zur Eigenleistung zu unterstützen, d.h. Hilfe zur Selbsthilfe. Erst wenn der Körper nicht mehr in der Lage ist, selbst zu laufen, in der Regel bei vitaler Indikation, muss die Krücke helfen; das heisst für die antibiotische Therapie: so wenig wie möglich, so viel wie nötig. 
Die Infektionskrankheiten bieten überhaupt ein treffendes Beispiel für die falsch angewandten Krücken und ihre Folgen: Das ungeübte Immunsystem wird mit dem Erreger nicht mehr fertig, der Körper erliegt ihm oder Rezidivierung und Chronifizierung sind die Folge. Zudem kommt es zur Züchtung resistenter Erreger - die Krücke ist durch zu häufigen Gebrauch abgenutzt, die Waffe ist stumpf geworden.

Man hat oft den Eindruck, als sei die Therapie der Infektionskrankheiten bei Ehrlichs Prinzip der «Therapia magna sterilisans» stehen geblieben. Die zweifellos vorhandenen Fortschritte durch die aktuelle Chemotherapie werden durch deren kritiklose Anwendung oft aufgehoben. Es gibt heute wieder nosokomiale, d.h. durch Keime im Krankenhaus verursachte, Infektionskrankheiten wie zu Semmelweis' Zeiten. Dagegen haben Kliniker wie Hoff schon vor einiger Zeit ausdrücklich auf das Unterstützungsprinzip bei der Behandlung der Infektionskrankheiten hingewiesen [7], und Höring hat davor gewarnt, den Bazillus als personifizierte Krankheit anzusehen [8].

Brechts Arzt behandelt eine Krankheit, die durch falsche Behandlung chronifizierte, dadurch zur Abhängigkeit führte und selbst zur Krankheitsursache wurde. Diesem Sachverhalt begegnen wir heute oft, denn die Indikation zur Ersatzleistung in der Therapie wird in Deutschland weit überschätzt. Häufig ist z.B. eine Arzneiverordnung für den Patienten nur eine Krücke, der er sich aus Bequemlichkeit bedient, weil es für ihn einfacher ist, eine Arznei zu schlucken, als seinen Lebensstil zu ändern, zumal ihm die negativen finanziellen Folgen von der Solidargemeinschaft abgenommen werden. Mit Recht wird die Passivisierung und Medikalisierung breiter Bevölkerungskreise beklagt. Wie oft ist die Begegnung mit dem Arzt selbst eine Ersatzleistung, an die sich der Patient, oft aus Vereinsamung, gewöhnt hat - Freud hat davor gewarnt -, oder der chirurgische Eingriff ohne zwingende Indikation, ohne den man auch - oder besser - helfen könnte. Die Kosten für die Behandlung selbstverschuldeter Krankheiten gehen in die Milliarden. Unsere Zeit ist in vieler Hinsicht ein Zeitalter des Ersatzes, was ich an anderer Stelle zu beschreiben versucht habe [9]. Ohne Zweifel hat auch Brecht die Erfahrung zu seinem Gedicht nicht allein aus der Medizin erhalten. Sein mehrmonatiges Medizinstudium und seine kurze Tätigkeit als Krankenpfleger 1918 dürften dazu kaum ausreichen. Sicher gehört auch eigenes Krankheitserleben zu diesen Erfahrungen. Er hat das Gedicht $1938 \mathrm{im}$ Exil in Dänemark geschrieben. Es mag verwundern, dass jemand, in dessen Werken das Helfen eine so bedeutende Rolle spielte, sich kritisch zum Helfen äussert. Hier ist nur anzumerken, dass ein Psychologe vom Range Brechts die Folgen einer falschen Hilfe, die das Gegenteil ihrer eigentlichen Absicht zur Folge hat, erkannte und davor warnen wollte. (Das Wort «Krücke» hatte immer einen negativen
Beiklang und darf heute in der Medizin nicht mehr verwendet werden.)

Brechts Arzt begann die Therapie mit dem Zerbrechen der Krücken. Die Naturheilkunde bezeichnet die Beseitigung krankmachender Abhängigkeiten als «Ordnungstherapie», als den ersten Schritt. Das ist heute schwierig, denn auf die Krücken zu verzichten, ist, wie gesagt, für viele Menschen - nicht nur für die Süchtigen - mit einem oft sehr negativen Absetzeffekt verbunden, mit einem Verzicht. Aber Brechts Kranker kam ja ausdrücklich mit dem Wunsch auf ärztliche Hilfe, um gesund zu werden und arztfrei leben zu können, was ihm durch die tatkräftige Hilfe des Arztes und dank seiner eigenen Behandlungstreue auch gelang.

Ebenso wie für die Ersatzleistung gilt auch für die Schonung das Prinzip: So wenig wie möglich, so viel wie nötig, denn jedes Zuviel engt die Funktion ein und muss später wieder aufgebaut werden. Hier bedarf es der ärztlichen Erfahrung in der Kenntnis der therapeutischen Mittel ebenso wie die der reparativen Möglichkeiten des Organs.

Zur vollen Wiederherstellung der Funktion bedarf es dann der Übung. «Geh, fall, kriech auf allen vieren!» ist allerdings ein Appell, den Brecht gewiss mit Recht auf andere Lebensbereiche bezieht. Er setzt voraus, dass der Genesende zu einem unter Umständen harten Übungsprogramm bereit sein muss. Die Funktionelle Therapie arbeitet hier mit Funktionsreizen in angepasster Dosierung, möglichst im beschwerdefreien Raum. Die Krankengymnastik z.B. ist zum Wiederaufbau der Funktion von Kreislauf und Bewegungsapparat - soweit diese es noch erlaubt - seit je integrierender Teil der Naturheilkunde. Hier muss um jeden Fussbreit Bodens gerungen werden und der Patient immer wieder zur Behandlungstreue ermahnt werden. Wie schwierig der Weg zur Gesundheit angesichts der Verlockungen zur Ersatzleistung sein kann, hat Brecht grossartig beschrieben: «Nur zuweilen, wenn ich Hölzer sehe, gehe ich für Stunden etwas schlechter».

Die Versuchung ist ebenso für den Arzt gegeben. Es sollte aber Ziel einer jeden Behandlung sein, den Patienten gesund zu machen, sodass eine ärztliche Behandlung nicht notwendig ist. Die gesellschaftliche Situation ist bekannt, die Heerscharen von Menschen Krücken anbietet und das verhindert. Die Worte «sozial» und «solidarisch» sind zu Schlagworten verkommen.

Brechts Arzt behandelte einen chronisch Kranken mit den Prinzipien der Naturheilkunde. Das wirft die Frage auf, ob und wie denn akute Krankheiten mit ihren Verfahren behandelt werden können. Die Antwort ist leider heute nötig und verlangt eine Zwischenbemerkung. Die Naturheilkunde arbeitet als Funktionelle Therapie mit dem Unterstützungsprinzip. Der umfangreiche Vorrat an entsprechenden Verfahren ist erst kürzlich ausführlich veröffentlicht worden [1]. Eigentlich beginnt sie im Haushalt 
der Familie, wo von dem früher sehr reichhaltigen Wissen heute oft nur noch die Wadenwickel beim fiebernden Kind erhalten geblieben sind. Früher kannte man unter anderem die ansteigende Serie vom Waden-, Gelenk-, Brust-, Leibbis zum Rumpfwickel, die Andampfungen, die ansteigenden Teilbäder und die gliedweisen Waschungen. Die Klinik kennt heute allenfalls noch die ansteigenden Unterarmbäder nach Hauffe-Schweninger, um die Venen punktionsfähig zu machen. Die historische Entwicklung, die dazu führte, kann an anderer Stelle nachgelesen werden. Beispielhaft dafür ist die Entwicklung in Berlin. Für die Naturheilkunde wurden hier 1919 eine Klinik, eine Poliklinik und ein Lehrstuhl für «Natürliche Heil- und Lebensweisen» errichtet. Die Klinik wurde geschlossen und die Poliklinik in den 1980er-Jahren in "Poliklinik für physikalische Medizin und Rehabilitation» umbenannt [10], nachdem der Begriff «Rehabilitation» in den 1970er-Jahren in die Medizin eingeführt worden war. Voraussetzung dazu war die Ergänzung des Begriffs «Krankheit» der kurativen Medizin durch den Begriff «Behinderung», der im Zuge der präventiven Medizin laufend an Bedeutung gewann (Sozialgesetzbuch IX). Dadurch wurde die Naturheilkunde mit der Bäderheilkunde verbunden, wo sie in der Regel zur Nachbehandlung akuter Krankheiten angewandt wird (man fährt heute zur Rehabilitation/Reha).

Ausser in den offiziellen Heilstätten der Sozialversicherung wird Naturheilkunde von zahlreichen privaten Sana- torien und Kuranstalten angeboten. Bestrebungen zu ihrer Wiederbelebung sind im Gange. So wurde das Berliner Immanuel-Krankenhaus als «Klinik und Hochschulambulanz für Naturheilkunde» der Universität angegliedert.

Doch noch einmal zurück: Brechts Anregung ist es zu verdanken, dass zu Pathologie und Diagnostik auch in der Therapie auf den Funktionsbegriff nachdrücklich hingewiesen wurde. Es war nötig, den Begriff «Funktionelle Therapie» als Terminus und Oberbegriff für alle Therapieverfahren einzuführen. Die Naturheilkunde hat darin ihren Platz als Verfahren, das die Unterstützung der Heilvorgänge als ihr Prinzip ansieht. Angesichts der Tatsache, dass sich die Arzneimittelausgaben der Gesetzlichen Krankenversicherung seit der Wiedervereinigung in Deutschland nahezu verdoppelt haben, 2009 die Summe von 28,5 Milliarden Euro erreicht wurde und die Kosten weiter ansteigen, ist die Feststellung keine Übertreibung, dass die konservative Therapie heute in den Händen der pharmazeutischen Industrie liegt. Die Heilkunde ist in den Sog des Konsumismus geraten, und die Menschen haben heute oft andere Motive, den Arzt aufzusuchen, als Brechts Kranker.

\section{Disclosure Statement}

Es bestehen keinerlei Interessenkonflikte.

\section{Literatur}

1 Kraft K, Stange R: Lehrbuch Naturheilverfahren. Stuttgart, Hippokrates, 2010.

2 Meyers kleines Konversationslexikon. Leipzig, Bibliografisches Institut, 1909, p 932.

3 «Der Naturarzt» / Zeitschrift des Deutschen Bundes der Vereine für Gesundheitspflege und für arzneilose Heilweise. Berlin, Wilhelm Möller, 1892
4 Brecht B: «Die Krücken»; in Brecht B: Werke. Grosse kommentierte Berliner und Frankfurter Ausgabe, Band 4: Gedichte 4. Frankfurt/M., Suhrkamp, 1993.

5 von Bergmann G: Funktionelle Pathologie. Berlin, Springer, 1936.

6 Ruda C: Zur Pathologie und Therapie der Funktion: «Die Heilkunst». München, Heilkunstverlag, 1963
7 Hoff F: Fieber, unspezifische Abwehrvorgänge, unspezifische Therapie. Stuttgart, Thieme, 1957.

8 Höring F: Klinische Infektionslehre. Berlin, Springer, 1948, p 1.

9 Ruda C: Der gefesselte Mensch, Neurose und Gesellschaft. Frankfurt/M., Ullstein, 1986.

10 Ruda C: Die Naturheilkunde in Berlin. Berliner Ärzteblatt 1999;112:426. 